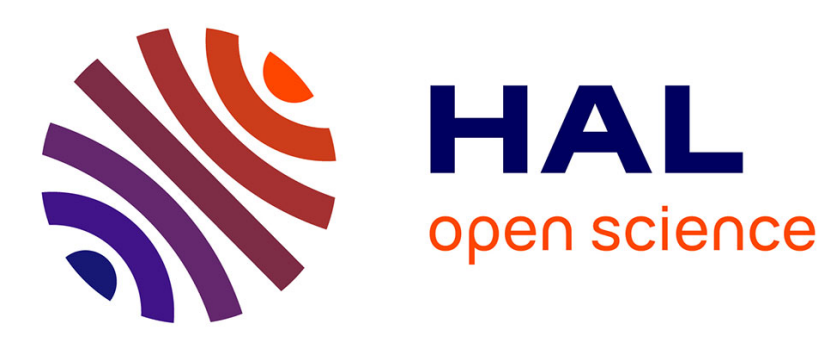

\title{
Multivariate single-subject analysis of short-term reorganization in the language network \\ Gesa Hartwigsen, Danilo Bzdok
}

\section{To cite this version:}

Gesa Hartwigsen, Danilo Bzdok. Multivariate single-subject analysis of short-term reorganization in the language network. Cortex, 2018, pp.4. 10.1016/j.cortex.2018.06.013 . hal-01824229

\section{HAL Id: hal-01824229 \\ https://hal.science/hal-01824229}

Submitted on 19 Jul 2018

HAL is a multi-disciplinary open access archive for the deposit and dissemination of scientific research documents, whether they are published or not. The documents may come from teaching and research institutions in France or abroad, or from public or private research centers.
L'archive ouverte pluridisciplinaire HAL, est destinée au dépôt et à la diffusion de documents scientifiques de niveau recherche, publiés ou non, émanant des établissements d'enseignement et de recherche français ou étrangers, des laboratoires publics ou privés. 
Dear author,

Please note that changes made in the online proofing system will be added to the article before publication but are not reflected in this PDF.

We also ask that this file not be used for submitting corrections. 


\section{Letter to the editor}

\section{Multivariate single-subject analysis of short-term reorganization in the language network}

Q3 Gesa Hartwigsen ${ }^{a, *}$ and Danilo Bzdok ${ }^{b, c, d, * * *}$

${ }^{a}$ Research Group Modulation of Language Networks, Department of Neuropsychology, Max Planck Institute for

Q1 Human Cognitive and Brain Sciences Leipzig, Leipzig, Germany

$\mathrm{b}$ Department of Psychiatry, Psychotherapy and Psychosomatics, Medical Faculty, RWTH Aachen, Aachen, Germany

c JARA-BRAIN, Jülich-Aachen Research Alliance, Germany

d Parietal Team, INRIA, Neurospin, Gif-sur-Yvette, France

\section{A R T I C L E I N F O}

Article history:

Received 3 February 2018

Reviewed 12 March 2018

Revised 7 April 2018

Accepted 26 June 2018

Action editor Peter Turkeltaub

Published online $\mathrm{xxx}$

Numerous functional neuroimaging studies in humans used univariate group analyses to identify brain areas that show increased activity across participants during specific tasks. However, there is increasing evidence that group-level analyses may obscure important parts of the signal response (Margulies, 2017; Poldrack, 2017). Here, we re-analyzed data from our recent study (Hartwigsen et al., 2017) that investigated functional reorganization in the language network. We show that across-voxel pattern-learning approaches are useful to isolate plastic changes in neural networks underlying cognitive functions at the individual subject level. Using predictive machine-learning tools to identify and exploit subject-specific neural activity patterns have been argued to become an important cornerstone of precision medicine in psychiatry and neurology (Arbabshirani, Plis, Sui, \& Calhoun, 2017; Bzdok \& Meyer-Lindenberg, 2018; Woo, Chang, Lindquist, \& Wager, 2017).
Functional reorganization is a key process for language recovery after lesions. However, current knowledge of plasticity in language networks is insufficient (Turkeltaub, 2015). Combining focal perturbations induced by transcranial magnetic stimulation (TMS) and FMRI in the healthy brain, we recently provided insight into the reorganization potential of language networks (Hartwigsen et al., 2017). Causal impairment of a key node for the processing of the meaning of words (semantic processing) in left angular gyrus (AG) entailed decreased semantic activity in a large network, including the targeted area. Despite this inhibition, there was no significant performance deterioration. Strikingly, this inhibition resulted in an upregulation of a neighboring network for phonological processing (processing of the sound of words), including left supramarginal gyrus (SMG) and adjacent superior parietal lobe (SPL). These regions were not engaged during semantic processing before TMS. This upregulation might have partially compensated for the disruption of the semantic network. In contrast, TMS over the phonological node in SMG resulted in strong inhibition of phonological regions and performance deterioration, without any compensatory upregulation of other areas. The difference in the activity patterns for both tasks might reflect the differential potential of brain networks for compensation of focal disruption that may be nonidentical in different individuals. Despite its role in phonological processes, SMG/SPL appears to be capable of supporting semantic processing when task demands increase (after a virtual lesion of $A G$ ). In contrast, there was no evidence for a supportive function of the AG after perturbation of SMG.

\footnotetext{
* Corresponding author. Research Group Modulation of Language Networks, Department of Neuropsychology, MPI for Human Cognitive Q2 and Brain Sciences, Stephanstr. 1a, D-04103 Leipzig, Germany.

** Corresponding author. Department of Psychiatry, RWTH, Aachen University, D-52074 Aachen, Germany.

E-mail addresses: hartwigsen@cbs.mpg.de (G. Hartwigsen), danilo.bzdok@rwth-aachen.de (D. Bzdok). 


\section{A predictive patterns of semantic responses $\quad B$ predictive patterns of TMS disruption \\ sham TMS \\ TMS over AG \\ TMS over AG > sham TMS}

subject 1

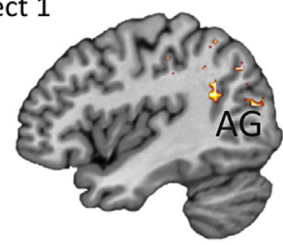

subject 2

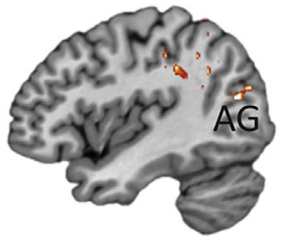

subject 3

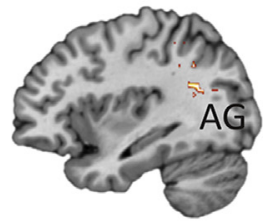

subject 4

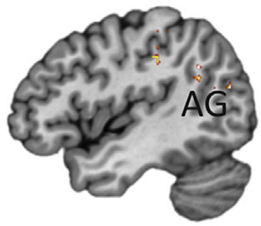

subject 5

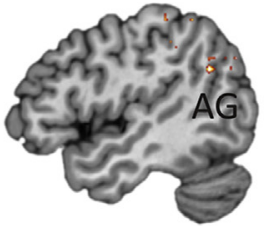

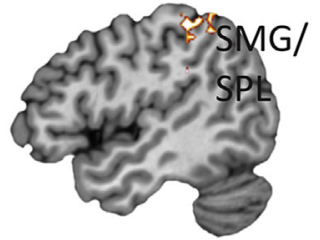
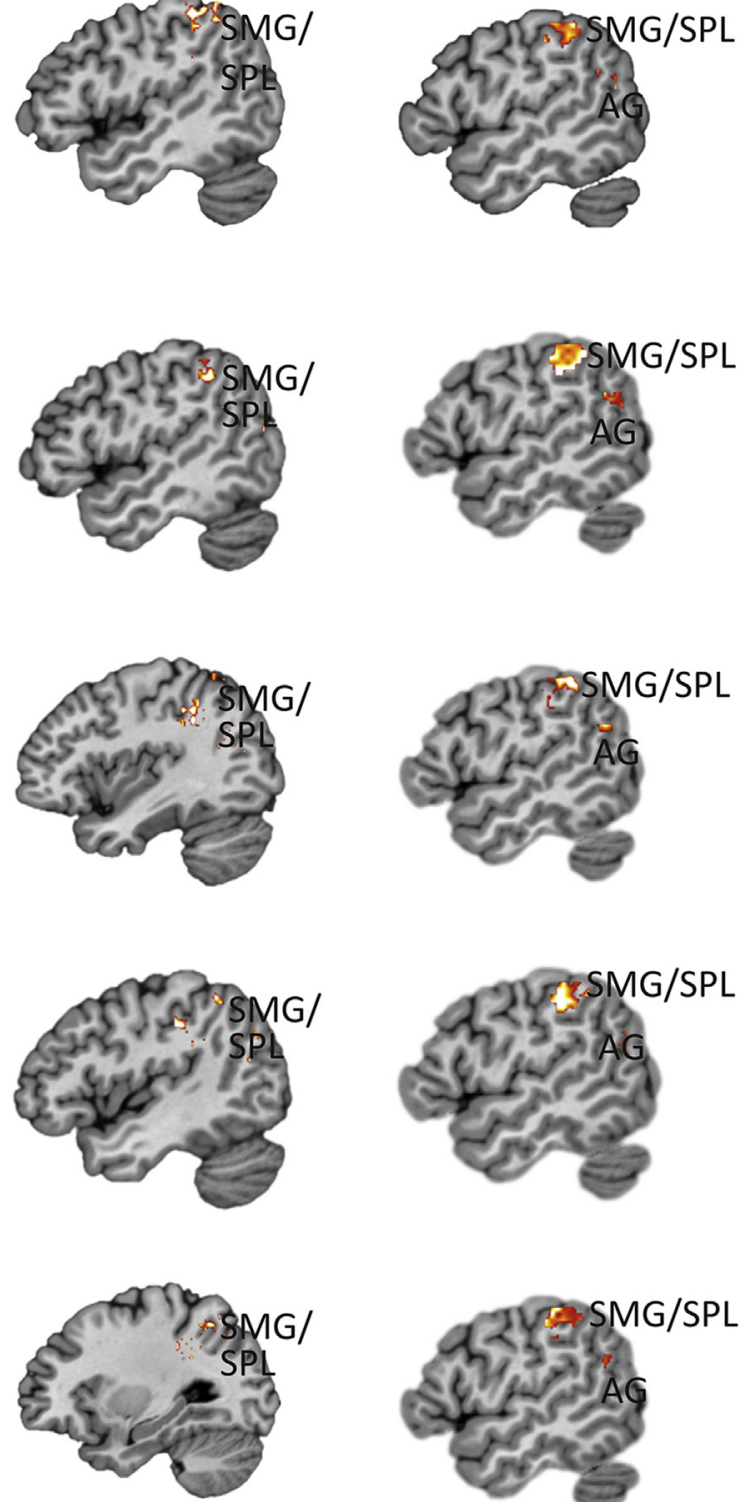
These findings were only significant at the group level. However, group analyses may limit the detail, specificity, and clinical utility of task-activation experiments (Gordon et al., 2017b). Specifically, certain organizational features of brain networks present in the individual were lost when averaging across groups (Braga \& Buckner, 2017). There is interest in predicting subject-specific changes in neural activity (Braga \& Buckner, 2017; Gordon et al., 2017a; Gordon et al., 2017b). Recent studies show increased use of sensitive multivariate measures to detect such effects (Bzdok, 2017; Haynes, 2015). Given its strength to detect changes in process-specific neural response, a multi-voxel pattern analysis (MVPA) should reveal intervention-specific effects on the single subject level (Haxby et al., 2001). MVPA allows for testing whether distributed activity patterns carry information about different stimulus types (Schomers \& Pulvermuller, 2016). To our knowledge, MVPA has never been used to detail TMS-induced changes in adaptive plasticity. To elaborate on our hypothesis that SMG might functionally compensate for disruption of AG, we performed two MVPA analyses on the individual subject level of the original data (Supplementary Information).

A first MVPA aimed at identifying neural activity patterns underlying semantic processing in left AG and SMG after placebo or effective TMS. This analysis addressed the following question: Which parts of the brain exhibit neural activity patterns that predict the presence of two semantic stimulus types (natural us man-made categories in our stimuli) after TMS of AG in a single individual? Our searchlight analysis (Kriegeskorte, Goebel, \& Bandettini, 2006) elucidated that after sham TMS, predominantly voxels located in AG predicted differences in activity, supporting its role in semantic processing (Binder, Desai, Graves, \& Conant, 2009). After effective TMS, discriminability focus shifted from AG to the phonological region in SMG (Fig. 1A \& SIFig. 1A).

The second MVPA addressed a distinct question: Which voxels can predict plasticity patterns across stimulus types with versus without TMS of AG? We used TMS type (effective us sham) as targeted conditions that were predicted. This analysis identified voxels that successfully distinguished TMSinduced changes in neural plasticity patterns during semantic processing. Results confirmed that effective TMS altered processing in AG. Outside of the TMS focus, the strongest effect was found in SMG (Fig. 1B \& SIFig. 1B). This confirms our hypothesis that plastic changes in SMG are reliably induced by TMS over AG.

Notably, the MVPA results were only informative at the individual subject level. Due to inter-individual variability, extracted predictive patterns were not robust at the group level. This finding further emphasizes the importance of single-subject analyses to map particularly fine-grained neural responses.

Both MVPAs strengthen our hypothesis of SMG-mediated compensation in single subjects after disruption of AG. The univariate results indicated that $A G$ perturbation led to compensatory changes in neural activity level or extent in SMG. The multivariate results show that AG perturbation also induces changes in neural activity patterns carrying semantic information. Both support the notion that AG perturbation induces compensatory processing in SMG. These results point to a generic mechanism of network plasticity, arguing for a flexible redistribution of function. Our results help to identify adaptive patterns of reorganization after a lesion to a cognitive core region. Particularly, multivariate approaches might inform future studies to select areas for the application of TMS to facilitate language recovery after brain lesions. Based on our findings, we speculate that facilitation of neighboring regions might help supporting language recovery.

Despite only 60 stimuli per semantic condition and the fact that neural patterns underlying cognitive processes tend to be subject-specific (Saygin et al., 2011), we successfully isolated predictive patterns in all subjects. Future studies may provide insight from larger data sets and optimized experimental designs. Such studies would advance the current knowledge of plasticity in cognitive networks and ultimately help to understand how the brain reorganizes after lesions to processspecific nodes. The combination of TMS and machinelearning statistics has the potential for new insight into plastic changes on the individual subject level that might be used for outcome prediction and therapeutic intervention in future personalized medicine.

\section{Supplementary data}

Supplementary data related to this article can be found at https://doi.org/10.1016/j.cortex.2018.06.013.

\section{R E F E R E N C E S}

Arbabshirani, M. R., Plis, S., Sui, J., \& Calhoun, V. D. (2017). Single subject prediction of brain disorders in neuroimaging: Promises and pitfalls. Neuroimage, 145(Pt B), 137-165. https:// doi.org/10.1016/j.neuroimage.2016.02.079.

Binder, J. R., Desai, R. H., Graves, W. W., \& Conant, L. L. (2009). Where is the semantic system? A critical review and metaanalysis of 120 functional neuroimaging studies. Cerebral Cortex, 19, 2767-2796.

Bludau, S., Bzdok, D., Gruber, O., Kohn, N., Riedl, V., Sorg, C., et al. (2016). Medial prefrontal aberrations in major depressive disorder revealed by cytoarchitectonically informed voxel-based morphometry. The American Journal of Psychiatry, 173, 291-298. https://doi.org/10.1176/ appi.ajp.2015.15030349.

Braga, R. M., \& Buckner, R. L. (2017). Parallel interdigitated distributed networks within the individual estimated by intrinsic functional connectivity. Neuron, 95(2), 457-471. https://doi.org/10.1016/j.neuron.2017.06.038. e455.

Bzdok, D. (2017). Classical statistics and statistical learning in imaging neuroscience. Frontiers in Neuroscience, 11, 543. https:// doi.org/10.3389/fnins.2017.00543. eCollection 2017. Review.

Bzdok, D., \& Meyer-Lindenberg, A. (2018). Machine learning for precision Psychiatry: Opportunities and challenges. Biological Psychiatry: Cognitive Neuroscience and Neuroimaging, 3(3), 223-230. https://doi.org/10.1016/j.bpsc.2017.11.007.

Gordon, E. M., Laumann, T. O., Adeyemo, B., Gilmore, A. W., Nelson, S. M., Dosenbach, N. U. F., et al. (2017a). Individualspecific features of brain systems identified with resting state functional correlations. Neuroimage, 146, 918-939. https:// doi.org/10.1016/j.neuroimage.2016.08.032.

Gordon, E. M., Laumann, T. O., Gilmore, A. W., Newbold, D. J., Greene, D. J., Berg, J. J., et al. (2017b). Precision functional 
mapping of individual human brains. Neuron, 95(4), 791-807. https://doi.org/10.1016/j.neuron.2017.07.011. e797.

Hartwigsen, G., Bzdok, D., Klein, M., Wawrzyniak, M., Stockert, A., Wrede, K., et al. (2017). Rapid short-term reorganization in the language network. Elife, 6. https://doi.org/10.7554/eLife.25964.

Haxby, J. V., Gobbini, M. I., Furey, M. L., Ishai, A., Schouten, J. L., \& Pietrini, P. (2001). Distributed and overlapping representations of faces and objects in ventral temporal cortex. Science, 293(5539), 2425-2430. https://doi.org/10.1126/ science.1063736.

Haynes, J.-D. (2015). A primer on pattern-based approaches to fMRI: Principles, pitfalls, and perspectives. Neuron, 87(2), 257-270.

Kriegeskorte, N., Goebel, R., \& Bandettini, P. (2006). Informationbased functional brain mapping. Proceedings of the National Academy of Sciences USA, 103(10), 3863-3868. https://doi.org/ 10.1073/pnas.0600244103.

Margulies, D. S. (2017). Unraveling the complex tapestry of association networks. Neuron, 95(2), 239-241. https://doi.org/ 10.1016/j.neuron.2017.07.006.
Poldrack, R. A. (2017). Precision neuroscience: Dense sampling of individual brains. Neuron, 95(4), 727-729. https://doi.org/

10.1016/j.neuron.2017.08.002.

Saygin, Z. M., Osher, D. E., Koldewyn, K., Reynolds, G., Gabrieli, J. D., \& Saxe, R. R. (2011). Anatomical connectivity patterns predict face selectivity in the fusiform gyrus. Nature Neuroscience, 15(2), 321-327. https://doi.org/10.1038/nn.3001.

Schomers, M. R., \& Pulvermuller, F. (2016). Is the sensorimotor cortex relevant for speech perception and understanding? An integrative review. Frontiers in Human Neuroscience, 10, 435. https://doi.org/10.3389/fnhum.2016.00435.

Turkeltaub, P. E. (2015). Brain stimulation and the role of the right hemisphere in aphasia recovery. Current Neurology and Neuroscience Reports, 15(11), 72. https://doi.org/10.1007/s11910015-0593-6.

Woo, C. W., Chang, L. J., Lindquist, M. A., \& Wager, T. D. (2017). Building better biomarkers: Brain models in translational neuroimaging. Nature Neuroscience, 20(3), 365-377. https:// doi.org/10.1038/nn.4478. 